\title{
Update from RCP Quality Improvement: Bringing together the experts to improve services
}

An essential part of any service improvement is to bring the right experts together. They can help to determine the current quality of care, where to focus improvement and what to measure to demonstrate if things have improved. Who could be more expert than patients, and yet true partnership with patients in service improvement remains the exception rather than the norm.

Ensuring true partnership with patients in decision making around care and services was a central feature of the Royal College of Physicians' (RCP's) Future Hospital Commission. ${ }^{1}$ The independent evaluation of the Future Hospital Programme highlighted the pivotal role of supporting patients to be within improvement teams in the success of the sites. ${ }^{2}$ Therefore, in the work of RCP Quality Improvement (RCPQI), we are ensuring that patient partnership is central.

The RCP's Patient and Carer Network (PCN) has been established for 15 years. ${ }^{3}$ This is led by patients, but with managerial support and a consultant physician as a patient involvement officer to champion not only the PCN but patient partnership more generally in the work that RCP and physician-led teams do. The PCN is now part of the RCP's Quality Improvement and Patient Safety Department, emphasising the substantial role it will increasingly play in our work and our influence, while continuing to work across the RCP.

Patient feedback comes in many forms and is used to inform improvement projects. For patient partnership in improvement to mature, it needs planning and leadership. Start with the use of complaints and routine NHS patient experience measurement questionnaires. Bespoke questionnaires for different services, structured interviews, focus groups, patient interviews by patient champions and public engagement, are used and connected by exemplars. ${ }^{4,5}$ Co-production, using improvement techniques such as experience-based design, and having some projects led by patients who have the skills to do so must be our goal (Fig 1). ${ }^{6}$

Within most of the quality improvement literature including patients as part of improvement teams is highlighted, but what does this mean and how easy is it? What is important is that teams working on improvement recognise the need to partner with patients and carers from the start of any work. Dependent on their local situations, the way this is done will vary based on what measures or interactions are already available. Having a patient and carer advocate as part of the core improvement team is necessary but this can be tokenistic and is not enough on its own. The individual advocate needs support to understand the role and how to maximise the benefit; they may need skill development, mentoring and support networks. They will need to link with other team members for patient feedback and partnership opportunities, including experience-based design, chairing patient panels, leading focus groups and informal conversations with patients. The rest of the team, and particularly the team lead, also need support to embrace and achieve the full benefits of the role of the patient and carer advocate. The PCN has published guidance on patient involvement in working groups which could be used and adapted by NHS organisations. ${ }^{7}$ There are examples within the UK where patients are receiving training in quality improvement methods so that they can be more active members of improvement teams. ${ }^{8}$
Fig 1. Hierarchy of patient partnership in service improvement.

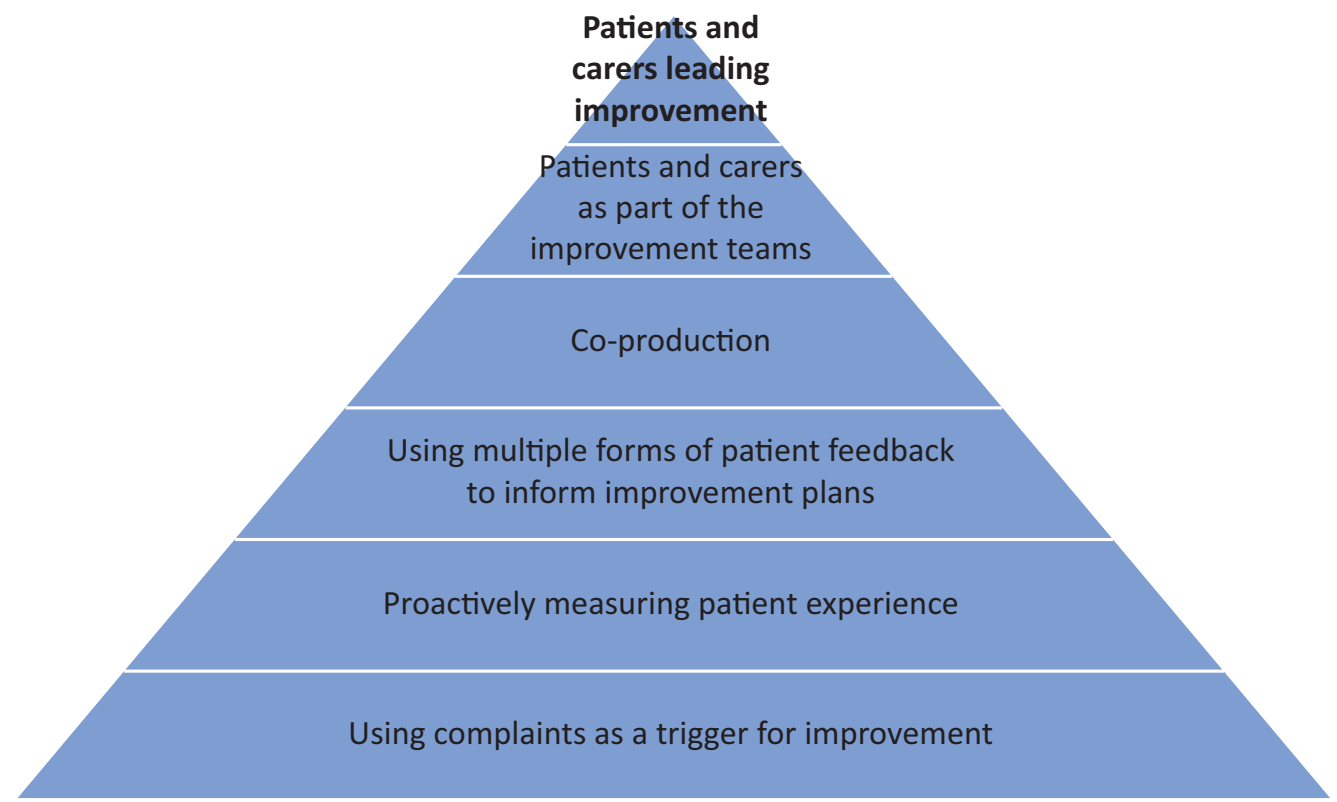


In RCPQI programmes, our breakthrough collaboratives have patient organisations as delivery partners and teams must include a patient advocate; support to develop patient involvement is incorporated in the learning sessions. Recent work on both ward rounds and outpatients has changed focus because of the input from the PCN and other patients and carers they have engaged with. For example, the importance to patients of seeing and being part of well organised team-based decision making on ward rounds, or the frustrations on the uncertainty of outpatient appointments and their disruption to daily living. Further work is required to understand how patients and carers can maximise their roles in improvement teams and we will be embarking on this work as well as training quality improvement practitioners in patient partnership approaches.

As quality improvement initiatives are submitted and published in Future Healthcare Journal we will be asking authors to describe patient involvement and its impact. Through these mechanisms we will continue to learn and develop practice and are more likely to improve outcomes that matter to patients.

Dr John Dean

Clinical director of quality improvement and patient safety, Royal College of Physicians

Dr Helen Gentles

Patient involvement officer, Royal College of Physicians

Laura Burling

Patient involvement adviser, Royal College of Physicians

\section{References}

1 Future Hospital Commission. Future hospital: Caring for medical patients. London: Royal College of Physicians, 2013. www.rcplondon. ac.uk/projects/outputs/future-hospital-commission

2 Future Hospital Programme. Delivering the future hospital. London: Royal College of Physicians, 2017. www.rcplondon.ac.uk/projects/ outputs/future-hospital-programme-delivering-future-hospital

3 Royal College of Physicians. Patient and Carer Network: Annual Report: April 2018 - June 2019. London: RCP, 2019. www.rcplondon.ac.uk/projects/outputs/patient-and-carer-network-annual-report-2018-19

4 The Health Foundation. Measuring patient experience. London: The Health Foundation, 2013. www.health.org.uk/publications/measuringpatient-experience

5 Taylor J. What about the patients? Experience is the weakest of the three arms of quality. HSJ 2019. www.hsj.co.uk/patient-safety/whatabout-the-patients-experience-is-the-weakest-of-the-three-arms-ofquality/7025528.article

6 The Point of Care Foundation. EBCD: Experience-based co-design toolkit. The Point of Care Foundation. www.pointofcarefoundation. org.uk/resource/experience-based-co-design-ebcd-toolkit

7 Royal College of Physicians. Working with lay representatives and patient involvement in RCP committees, boards and projects. London: RCP, 2019. www.rcplondon.ac.uk/file/13689/download?token=AfbusZ3a

8 Broad J. Q Exchange project patients are equal partners in quality improvement goes live. Q 2019. https://q.health.org.uk/blog-post/qexchange-project-patients-are-equal-partners-in-quality-improvementgoes-live

\section{Supervising quality improvement projects} A guide for supervisors

\begin{abstract}
This online resource has been developed by the Royal College of Physicians (RCP) to help clinicians from any specialty to identify learning opportunities relating to quality improvement, to support and guide their trainees throughout their quality improvement projects.
\end{abstract}

For more information, please email: elearning@rcplondon.ac.uk

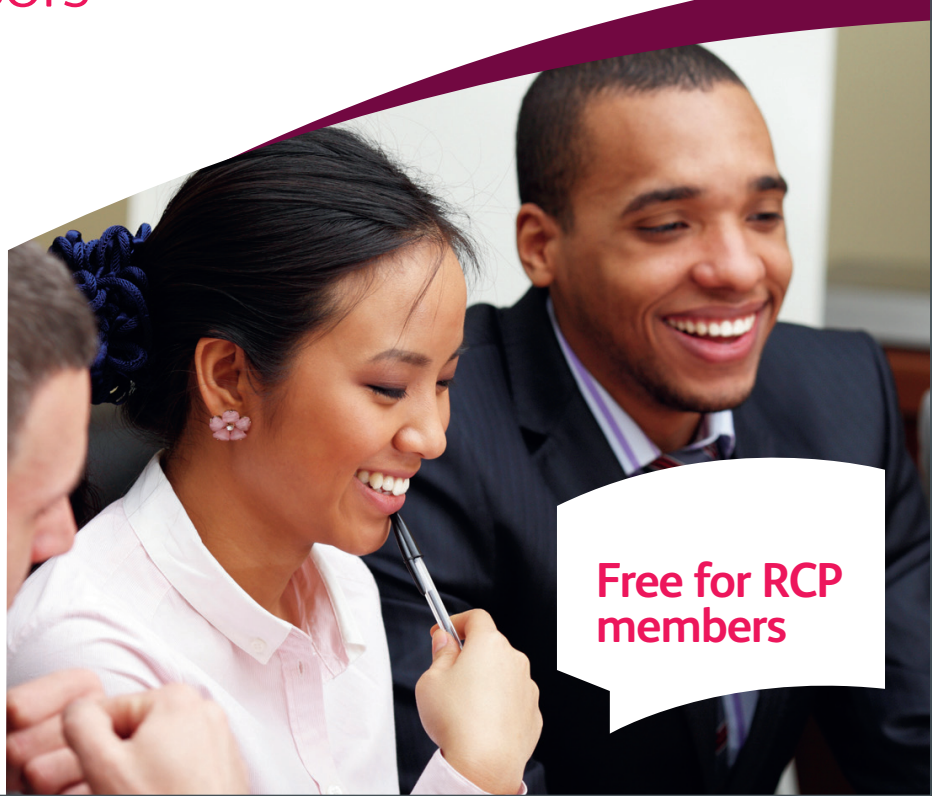

